\title{
Emergência de Relações Numéricas em Pré-Escolares
}

\author{
Mariana Morais Miccione* \\ Grauben José Alves de Assis \\ Universidade Federal do Pará, Belém, PA, Brasil \\ João dos Santos Carmo \\ Universidade Federal de São Carlos, São Carlos, SP, Brasil
}

\begin{abstract}
RESUMO
O paradigma das relações ordinais subsidia interpretação e análise de repertórios comportamentais envolvidos na aquisição de comportamentos ordinais acadêmicos. Três crianças com idades entre 4 a 5 anos participaram de dois estudos utilizando-se o procedimento de ensino informatizado por sobreposição de pares de estímulos. Na etapa 1 investigou-se a formação de relações ordinais após o ensino de duas sequências, numerais de 1 a 6 e suas quantidades. Todos formaram relações transitivas e classes ordinais. A etapa 2 verificou a emergência dessas relações ordinais sob controle condicional, na modalidade auditiva-visual. Todos formaram relações transitivase não houve formação de classes ordinais. Os resultados foram discutidos em termos de maior compreensão das variáveis do responder ordinal sob a modalidade auditiva-visual em pré-escolares.
\end{abstract}

Palavras-chave: relações ordinais; controle condicional; pré-escolares; repertórios acadêmicos.

\section{ABSTRACT}

\section{Emergence of Numeric Relations in Preschoolers}

The paradigm of ordinal relations subsidizes interpretation and analysis of behavioral repertoires involved in the acquisition of ordinal academic behaviors. Three children aged 4 to 5 years participated in two studies using a computerized teaching procedure for overlapping pairs of stimuli. The first step investigated the formation of ordinal relations after teaching two sequences, 1 to 6 numerals and their quantities. All formed transitive relations and ordinal classes. The second step examined the emergence of these ordinal relations under conditional control in auditory-visual modality. All formed transitive relations and there was no formation of ordinal classes. The results were discussed in terms of greater understanding of ordinal response variables in auditory-visual modality in preschoolers.

Keywords: ordinal relations; conditional control; preschoolers; academic repertoires.

A produção de sequências comportamentais é um capítulo importante na Análise Experimental do Comportamento. Os primeiros estudos sobre produção de sequências utilizavam o procedimento de encadeamento de respostas a fim de investigar as variáveis cruciais envolvidas na aquisição dessas sequências. Esse modelo, proposto por Skinner (1938/1991), produziu uma série significativa de estudos sobre cadeias de respostas e foi bastante útil na descrição da cadeia comportamental como sendo um fluxo de ações relacionadas a estímulos que exercem a dupla função de reforçar uma primeira resposta e sinalizar a ocasião para emissão de uma segunda resposta, em sequência. A rigor, o encadeamento inicia com o estabelecimento de uma discriminação simples, cujo reforçador tornase também estímulo discriminativo para a resposta seguinte, estendendo-se essa operação até se chegar à última resposta da cadeia, que produzirá o reforçador final. A utilidade desse modelo foi verificada tanto no ensino de repertórios acadêmicos básicos, como a contagem em matemática e a aquisição de linguagem verbal (Staats \& Staats, 1963), quanto no ensino de 
atividades de vida diária para indivíduos com deficiência intelectual (Hur \& Osborne, 1993). Com base nesses achados, uma ampla área de aplicação foi apresentada por Spradlin (1999), e estudos comparativos sobre variações nas técnicas de encadeamento até hoje são produzidos (Bancroft, Weiss, Libby, \& Ahearn, 2011).

Apesar de ser um modelo bastante útil, a produção de sequências por meio das diferentes variantes do encadeamento não oferece suporte à explicação da formação de sequências cujos elementos sejam intercambiáveis e, portanto, equivalentes entre si. Em outras palavras, o modelo de encadeamento não prevê que elementos de sequências podem se tornar equivalentes quanto à posição e ordem em que se encontram na sequência. Identificar as variáveis cruciais que nos ajudam a prever e controlar a formação de sequências ordinais equivalentes, e, portanto, a emergência de sequências novas, nunca antes diretamente treinadas, foi a questão investigada em um estudo seminal de Green, Stromer e Mackay (1993).

Nesse estudo, Green et al. (1993) partiram do modelo de equivalência de estímulos (Sidman \& Tailby, 1982) para analisarem a produção de sequências novas cuja ordem é variável de controle relevante sobre a formação de classes ordinais. Em linhas gerais, a proposta lançada por Green et al. (1993), concernente à compreensão da produção de relações ordinais, baseou-se no exame da adequação das noções de cadeia simples e discriminação condicional sobre os desempenhos engendrados a partir de contingências que estabelecem a produção de sequências. De acordo com esses autores, as relações entre os estímulos de uma mesma sequência e entre diferentes sequências poderiam ser documentadas a partir da realização de testes comportamentais que efetivassem as propriedades definidoras de uma relação de ordem da(s) sequência(s) ensinadas(s) (Stevens, 1951): irreflexividade, assimetria, transitividade e conectividade.

Considera-se uma relação como irreflexiva aquela relação ordinal que não se mostra reflexiva, isto é, na qual um elemento não pode ser seguido por ele mesmo. Portanto, a relação $\mathrm{A} 1 \rightarrow \mathrm{A} 1^{1}$ não é verdadeira. $\mathrm{A}$ propriedade da assimetria postula que uma relação ordinal é unidirecional; logo, A2 $\rightarrow$ A3 é uma relação verdadeira, mas o oposto $\mathrm{A} 3 \rightarrow \mathrm{A} 2$ não. $\mathrm{A}$ transitividade é observada quando dois pares de estímulos adjacentes dentro de uma sequência relacionam-se e deri- vam num terceiro par não adjacente; assim, A2 $\rightarrow$ A3 e $\mathrm{A} 3 \rightarrow \mathrm{A} 4$, então A2 $\rightarrow$ A4 é correta, na ausência do A3. Finalmente, uma relação apresenta a propriedade de conectividade se seus elementos se relacionaram em ordem consistente aos pares em todas as prováveis combinações; se $\mathrm{A} 1 \rightarrow \mathrm{A} 2 \rightarrow \mathrm{A} 3$, então $\mathrm{A} 1 \rightarrow \mathrm{A} 2$, $\mathrm{A} 2 \rightarrow \mathrm{A} 3, \mathrm{~A} 1 \rightarrow \mathrm{A} 3$ e A2 $\rightarrow \mathrm{A} 3$.

No que tange ao treino, o modelo de Green et al. (1993) proporciona a programação de dois tipos de procedimentos de ensino de repertórios sequenciais: por encadeamento (chaining) e via pares de estímulos sobrepostos (overlapping two-stimulus sequences). $\mathrm{O}$ ensino por encadeamento requer que os estímulos sejam acrescentados gradativamente, formando uma determinada sequência (e.g. $\mathrm{A} 1 \rightarrow \mathrm{A} 2, \mathrm{~A} 1 \rightarrow \mathrm{A} 2 \rightarrow \mathrm{A} 3$, $\mathrm{A} 1 \rightarrow \mathrm{A} 2 \rightarrow \mathrm{A} 3 \rightarrow \mathrm{A} 4, \quad \mathrm{~A} 1 \rightarrow \mathrm{A} 2 \rightarrow \mathrm{A} 3 \rightarrow \mathrm{A} 4 \rightarrow \mathrm{A} 5) . \quad \mathrm{O}$ ensino via pares de estímulos adjacentes sobrepostos requer a apresentação de uma sequência (e.g., $\mathrm{A} 1 \rightarrow \mathrm{A} 2, \mathrm{~A} 2 \rightarrow \mathrm{A} 3, \mathrm{~A} 3 \rightarrow \mathrm{A} 4, \mathrm{~A} 4 \rightarrow \mathrm{A} 5)$ de modo que todos os membros das sequências ensinadas só apareçam juntos nos testes (Holcomb, Stromer, \& Mackay, 1997; Souza, Assis, \& Magalhães, 2005; Stromer \& Mackay, 1993; Verdu, Souza, \& Lopes Júnior, 2005).

Diante desse panorama, um crescente conjunto de estudos passou a apresentar dados experimentais resultantes dessa proposta. A seguir serão descritos e discutidos alguns que mantêm estreita relação com os objetivos do presente relato de pesquisa.

Souza et al. (2005) conduziram dois estudos com o objetivo de demonstrar a efetividade do modelo experimental de relações ordinais sobre o repertório numérico em crianças surdas. O primeiro estudo avaliou os efeitos do procedimento de ensino por sobreposição, no qual os participantes deveriam responder a sequências de quatro pares de estímulos sobrepostos. Os desempenhos observados nos testes subsequentes de transitividade e conectividade foram satisfatórios e estenderam aqueles apresentados na linha de base. No Estudo 2, outras crianças surdas foram expostas ao mesmo procedimento de ensino e testes, porém com o acréscimo de testes de generalização com estímulos do ambiente natural e reexposição aos testes de conectividade. Os resultados mostraram que esses participantes responderam na primeira tentativa, tanto nos testes de generalização como nos retestes. Dessa feita, os dados confirmaram a eficiência do procedimento de ensino adotado, evidenciando que a relação entre estímulos equivalentes pode ocorrer mesmo fora dos 
parâmetros formais de emparelhamento com o modelo (Assis, Corrêa, Souza, \& Prado, 2010; Assis, Magalhães, Monteiro, \& Carmo, 2011; Verdu et al., 2005; Stromer \& Mackay, 1993; Holcomb et al., 1997).

Diante dos resultados das pesquisas citados anteriormente, alguns autores investigaram o responder ordinal pautando-se na possibilidade de acrescentar mais um membro nas contingências de sobreposição de estímulos e de teste, a fim de se ampliar a contingência de análise pelo estabelecimento do controle condicional.

Souza, Assis, Magalhães e Prado (2008) ensinaram cinco crianças surdas a produzir sequências de estímulos (Conjuntos A, B, C e D) por meio do procedimento desobreposição de estímulos sob controle condicional. Na presença da cor verde, o participante deveria ordenar de forma crescente os estímulos e, na presença da cor vermelha, de forma decrescente. Todos os participantes atenderam ao critério de acerto na fase de ensino. Testes avaliaram a formação de relações transitivas intraconjunto, de conectividade, de transferência de funções e de generalização. Todos os participantes responderam de acordo com os parâmetros experimentais planejados na maioria das tentativas. Segundo os autores, os dados encontrados confirmam a eficiência do procedimento em produzir a intercambialidade dos membros das sequências em função de suas posições ordinais mesmo sob controle condicional (Souza \& Assis, 2005).

Mais recentemente, Souza, Magalhães, Assis e Goulart (2010) estenderam ainda mais a contingência de análise ao investigarem a emergência de relações ordinais sob controle contextual em surdos. No estudo de Souza et al. (2010), a contingência diante do estímulo condicional "cor verde" ou "cor vermelha" seguia uma direção de seleção diferente dependendo se o estímulo contextual fosse "círculo" ou "triângulo". Cinco crianças surdas com história experimental em discriminações simples e condicional participaram do estudo. Todos os participantes atingiram o critério de acerto para a fase de ensino e responderam aos testes de transitividade e conectividade. Os autores discutem os resultados pontuando a importância da experiência prévia em contingências de discriminação simples e condicional sobre a emergência de classes ordinais, sob controle contextual.

Nos estudos descritos até aqui, a relação entre os estímulos discriminativos, condicionais e/ou contex- tuais foram apenas na modalidade visual-visual. Não consta na literatura sobre o responder ordinal, resultados de estudos experimentais na modalidade auditivovisual utilizando-se o procedimento por sobreposição de pares de estímulos. Sob distinta perspectiva metodológica, a do emparelhamento ao modelo, Green (1990) investigou a produção de relações de equivalência entre estímulos na modalidade auditivo-visual. O objetivo foi comparar o desempenho de cinco crianças com deficiência intelectual moderada na formação de classes de estímulos equivalentes, nas modalidades visual-visual e auditivo-visual. Expostos ao procedimento de emparelhamento ao modelo, os participantes foram ensinados a relacionar pares de estímulos nessas duas modalidades, obedecendo à ordem de exposição. Para os participantes que receberam primeiramente o treino na modalidade auditivo-visual, as relações de equivalência emergiram mais rapidamente. Dessa forma, esse estudo mostrou um padrão preponderante de aprendizagem nessa população em função da modalidade dos estímulos (ver também Sidman, Wilson-Morris, \& Kirk, 1986; Smeets \& Barnes-Holmes, 2005).

Face ao exposto, o presente relato de pesquisa destaca-se por apresentar os efeitos da utilização do procedimento por sobreposição de pares estímulos sobre a formação de classes ordinais com propriedades relacionais. Esse arranjo experimental é composto por contingências cujos estímulos discriminativos são disponibilizados simultaneamente, aos pares, diferentemente ao observado nas contingências dos estudos anteriormente citados, os quais envolvem a ordenação de pares de estímulos (condicional e discriminativo) dispostos sucessivamente, no formato de escolha de acordo com o modelo (matching to sample).

Dessa forma, o objetivo deste trabalho foi investigar a produção de sequências com base na análise experimental das relações ordinais em crianças préescolares, utilizando-se estímulos nas modalidades visual-visual e visual-auditiva.

\section{MÉTODO}

\section{Participantes}

Três crianças pré-escolares de ambos os gêneros, na faixa etária entre 4 anos e 2 meses e 5 anos e 8 meses de idade, que frequentavam o quinto ciclo de uma escola pública de Educação Infantil. Conforme 
Resolução 196/96 do Conselho Nacional de Saúde, um Termo de Consentimento Livre e Esclarecido foi assinado pelos pais dos alunos, autorizando a participação dos mesmos neste estudo. O projeto possui o número de protocolo 080/09 junto ao Comitê de Ética em Pesquisa em Seres Humanos.

\section{Ambiente Experimental e Equipamento}

As sessões experimentais ocorreram na sala de aula dos participantes, fora do horário de aula.

Um microcomputador (notebook) com sistema operacional Windows XP Professional, com processador Pentium III (Intel) e um monitor de 17" foi usado para a coleta dos dados comportamentais. O programa PROLER na versão 6.4 (Assis \& Santos, 2010) foi usado para apresentação dos estímulos e registro das respostas dos participantes. Esse programa não dispo- nibilizava o recurso de tela sensível ao toque, sendo simulado pela experimentadora pelo manuseio do mouse sobre os estímulos selecionados. Nas fases do Pré-teste e Pós-testes, utilizou-se cartelas com estímulos impressos feitas em papel cartão e peças de madeira em diferentes formas. As sessões experimentais aconteceram durante 3 meses, diariamente, com duração média de 30 minutos.

\section{Estímulos}

Dois conjuntos com seis estímulos foram utilizados (Figura 1). O conjunto "A" é composto pelos numerais cardinais de 1 a 6 . O conjunto " $\mathrm{B}$ ", por figuras não representacionais indicadoras das numerosidades de 1 a 6 em quatro disposições espaciais e em cinco tamanhos diferentes.

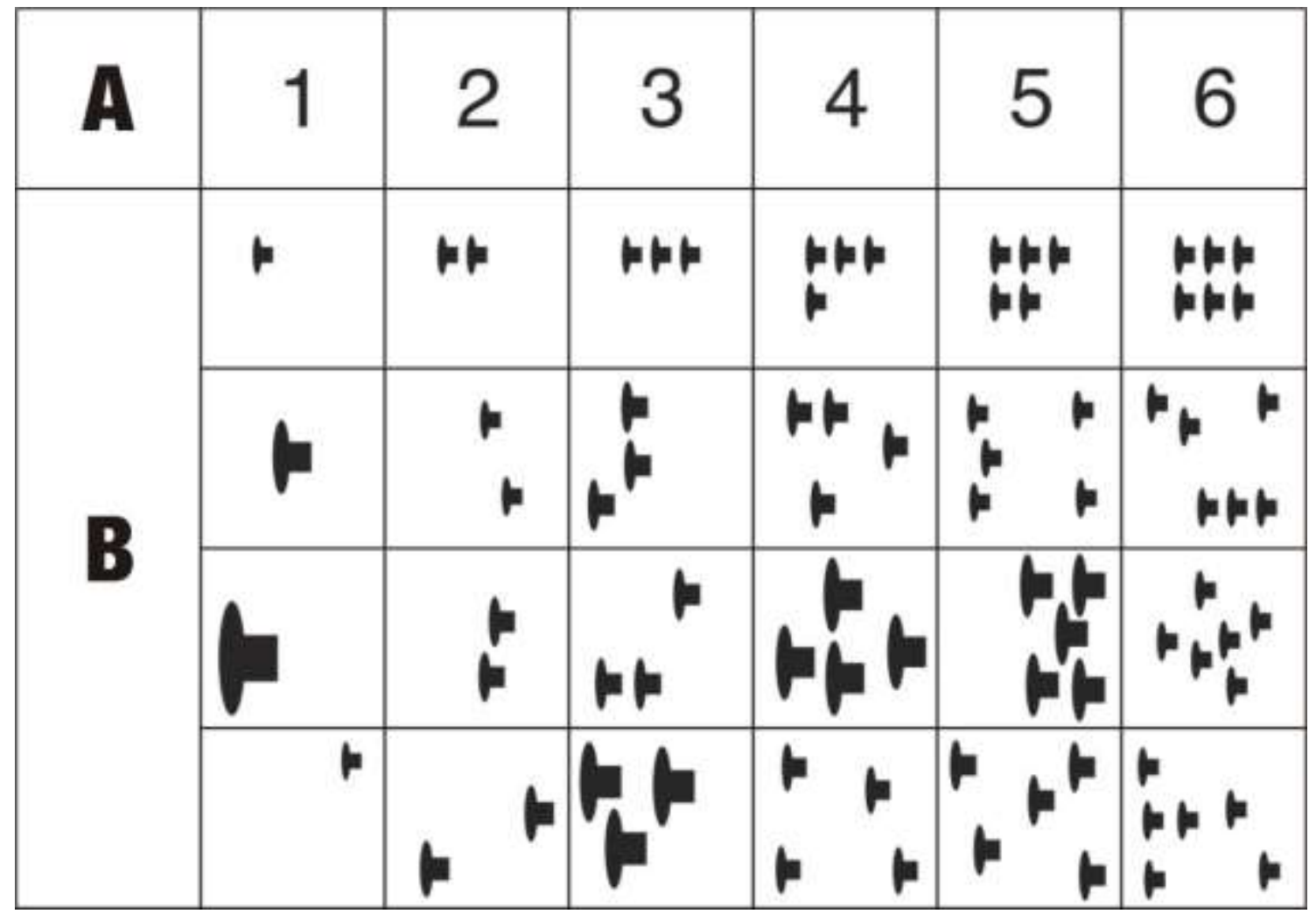

Figura 1. Conjunto de Estímulos “A” e "B".

\section{Procedimento}

Pré-testes. Um conjunto de tarefas não informatizadas foi programado para verificar a linha de base dos participantes.
Bloco 1 - Matching to sample arbitrário ordinal com dígitos na modalidade visual/visual: seis tentativas foram apresentadas ao participante da seguinte forma. Primeiramente, a experimentadora apresentava como modelo uma cartela $(4 \mathrm{~cm} \times 4 \mathrm{~cm})$, contendo 
um dígito (de 1 a 6 ) impresso juntamente com mais três cartelas contendo outros dígitos, como comparações. Em seguida, era solicitado ao participante que apontasse, dentre as três opções de escolha dispostas lado a lado, ao dígito que ocupasse a posição anterior ou posterior em relação ao modelo apresentado (localizado acima das escolhas). A instrução seguiu o padrão "Me diga qual desses números vem antes desse?" ou "Me diga qual desses números vai depois desse?". Os dígitos foram dispostos de modo a facultar apenas uma escolha, por exemplo, diante do dígito 3 como modelo, se solicitado ao participante qual dígito fosse depois dentre as opções de dígitos 1, 2 e 4, somente a terceira escolha poderia ser considerada correta.

Bloco 1.1 - Matching to sample arbitrário ordinal com blocos lógicos na modalidade visual/visual: aqui a lógica seguiu a mesma do Bloco 1. A instrução era "Me diga qual desses montinhos tem (mais/menos) que esse?".

Bloco 2 - Nomeação: as mesmas cartelas usadas no Bloco 1 foram apresentadas em seis tentativas aleatórias. A cada apresentação era solicitado ao participante que dissesse o nome de cada dígito após a instrução "Que número é esse?".

Bloco 3 - Matching to sample arbitrário com blocos lógicos na modalidade auditivo/visual: distribuído em seis tentativas a experimentadora apresentava como modelo a instrução oral, por exemplo, "Me diga em qual desses montinhos tem três pecinhas?" juntamente com três conjuntos de elementos dispostos lado a lado contendo três diferentes numerosidades, inclusive a equivalente à numerosidade ditada. $\mathrm{O}$ participante deveria apontar ao conjunto de peças que correspondesse ao modelo.

As respostas foram anotadas em um formulário pela pesquisadora. Não houve consequências diferenciais; respostas corretas e erradas produziam o encerramento da tentativa. Os participantes que totalizaram o escore de $50 \%$ ou menos de acertos em todos os blocos foram selecionados para a pesquisa.
Ao término de todas as sessões da pesquisa o participante poderia escolher dentre as seguintes opções: (a) adquirir um brinde nas categorias de brinquedo, enfeites de cabelo, adesivos, jogo de cartões ou material escolar; (b) desenhar livremente com giz de cera; (c) engajar-se no programa de computador Purble Place que continha três jogos, Purple pairs (jogo da memória), Comfy Cakes (montagem sequencial de bolos) e Purble Shop (MTS de identidade com modelo oculto). A verificação de preferência desses reforçadores foi realizada continuamente no decorrer das sessões.

\section{Fase Experimental - Programa PROLER:}

Procedimento Geral. Os participantes foram posicionados à frente ao microcomputador e a pesquisadora ficava ao seu lado direito monitorando a sessão experimental. Cada sessão era individualizada.

Após o consentimento dos participantes o programa começava a rodar. Cada tentativa iniciava com a apresentação de um quadrado localizado na parte superior da tela (ver Figura 2). A pesquisadora posicionava o cursor do mouse sobre esse quadrado e um click dava início à tentativa. Como consequência, eram apresentados simultaneamente dois estímulos visuais na "área de escolha" que deveriam ser selecionados.

Durante a fase de ensino, havia reforçamento diferencial. A consequência para acertos era combinada. Juntamente com as imagens de desenhos infantis apresentadas pelo computador, a experimentadora elogiava os participantes (e.g., "Parabéns, você acertou!"). Para os erros, a consequência era o escurecimento da tela por 1s (Time out). Nesses casos, os mesmos estímulos eram apresentados nas mesmas posições da tentativa anterior. Durante os testes, não havia consequência diferencial, uma tela em branco ao término da tentativa era apresentada. A matriz com a "área de escolha" e "área de construção" pode ser visualizada na Figura 2. 


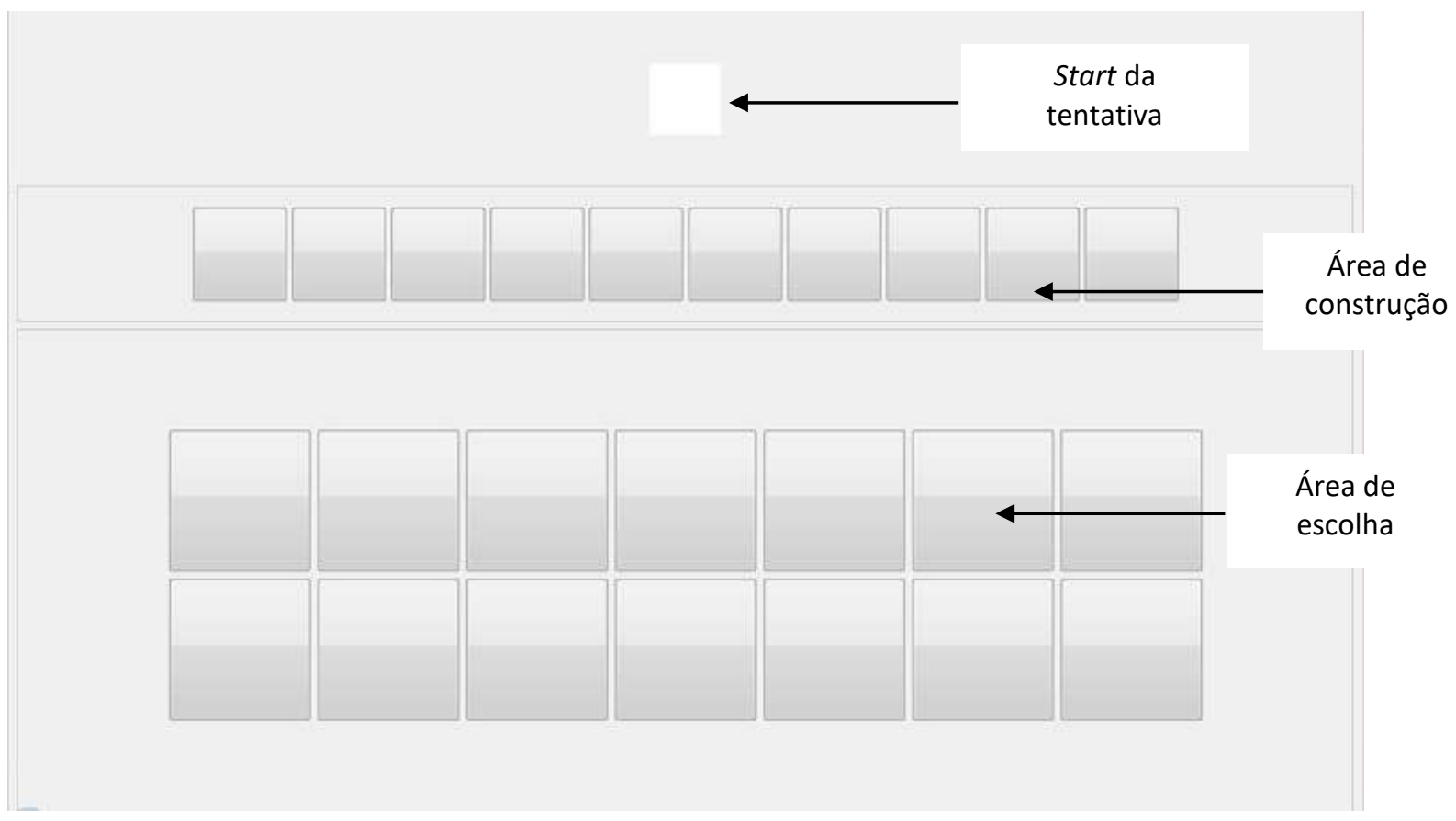

Figura 2. Matriz da tela de uma tentativa com as áreas de start, de construção da sequência e de escolha, onde os estímulos eram apresentados.

\section{Etapa 1}

Ensino de sequências simples por sobreposição. O participante deveria responder ordinalmente a cinco pares de estímulos ( $\mathrm{S}^{\mathrm{D}} \mathrm{s}$ compostos) sobrepostos (overlapping two stimulus sequences) nos quais o membro comum exercia, inicialmente, a função de segundo e, depois, de primeiro $(\mathrm{B} 1 \rightarrow \mathrm{B} 2 ; \mathrm{B} 2 \rightarrow \mathrm{B} 3 ; \mathrm{B} 3 \rightarrow \mathrm{B} 4$; $\mathrm{B} 4 \rightarrow \mathrm{B} 5$; B5 $\rightarrow$ B6).

Dois estímulos (B1 e B2) eram apresentados em duas das "janelas" da "área de escolha", em posições aleatórias. O participante deveria tocar primeiro em B1 e depois em B2. Ao tocar B1, o mesmo deslocavase para a "área de construção", permanecendo no primeiro dos 10 quadrados da esquerda para a direita. Ao tocar B2, o segundo estímulo também se deslocava para a área de construção, permanecendo ao lado direito de B1, no segundo quadrado da esquerda para a direita. As instruções seguiram o padrão "Escolha as duas figuras, uma após a outra" ou "Agora você vai continuar jogando. Você tem que apontar uma figura e depois a outra. Você entendeu?".

O critério de acerto era a formação correta de cada par de estímulos por três vezes consecutivas sem erro em até no máximo 10 tentativas, passando para a res- pectiva sonda (uma tentativa não sinalizada similar à tentativa de ensino, com os estímulos localizados em nova disposição e sem reforçamento diferencial). Sua inclusão teve a finalidade de preparar os participantes para as fases de testes, nas quais, sem nenhum tipo de sinalização de que não haveria consequenciação, as respostas não foram reforçadas diferencialmente. Caso o participante não respondesse a esse tipo de tentativa era reapresentado mais uma vez um bloco de ensino com o par não respondido seguido pela sonda.

Após alcançar o critério de acerto no ensino e na sonda, uma nova relação era ensinada, mantendo-se a última figura do par anterior (i.e., $\mathrm{B} 2 \rightarrow \mathrm{B} 3$ ) e, da mesma forma, era apresentada a respectiva tentativa de sonda. Nesse caso, na área de construção o estímulo B2 ocupava a primeira janela disponível e B3 a segunda. Após o ensino dos cinco pares sobrepostos $(\mathrm{B} 1 \rightarrow \mathrm{B} 2 ; \mathrm{B} 2 \rightarrow \mathrm{B} 3$; B3 $\rightarrow \mathrm{B} 4 ; \mathrm{B} 4 \rightarrow \mathrm{B} 5$; B5 $\rightarrow \mathrm{B} 6), \mathrm{um}$ bloco de sonda de verificação com reforço apresentava esses pares por meio de cinco tentativas aleatórias. O critério desse bloco era de $80 \%$ de acertos (quatro tentativas). Se não alcançado, um bloco de revisão de linha de base com critério de acerto de duas vezes consecutivas para cada par era apresentado. $\mathrm{O}$ mesmo procedimento de ensino anteriormente descrito foi adotado com os estímulos do Conjunto "A". 
Resultados nos testes de substituição encontrados por Miccione, Assis e Carmo (2012) mostraram que a totalidade dos erros apresentados por quatro dos cinco participantes se concentraram nas tentativas em que o primeiro estímulo a ser selecionado era do Conjunto B. Esses dados sugerem ter havido controle pela ordem de apresentação dos conjuntos na fase de ensino, isto é, primeiro o A, segundo o B. Dessa forma, programou-se o presente experimento no qual houve a inversão da apresentação dos conjuntos, isto é, primeiro o "B" e depois o "A".

Teste de transitividade. Consistiu na apresentação, dentre 10 pares possíveis, de cinco pares nãoadjacentes intra-sequência em três blocos: $\mathrm{B} 4 \rightarrow \mathrm{B} 6$, $\mathrm{B} 1 \rightarrow \mathrm{B} 5, \mathrm{~B} 1 \rightarrow \mathrm{B} 3, \quad \mathrm{~B} 3 \rightarrow \mathrm{B} 5, \mathrm{~B} 2 \rightarrow \mathrm{B} 5-\mathrm{B} 1 \rightarrow \mathrm{B} 4$, $\mathrm{B} 2 \rightarrow \mathrm{B} 6, \mathrm{~B} 1 \rightarrow \mathrm{B} 3, \mathrm{~B} 4 \rightarrow \mathrm{B} 6, \mathrm{~B} 3 \rightarrow \mathrm{B} 6-\mathrm{B} 1 \rightarrow \mathrm{B} 3$, $\mathrm{B} 2 \rightarrow \mathrm{B} 4, \mathrm{~B} 1 \rightarrow \mathrm{B} 5, \mathrm{~B} 3 \rightarrow \mathrm{B} 6, \mathrm{~B} 3 \rightarrow \mathrm{A} 5$. A seleção dos blocos era feita no momento da atividade. $\mathrm{O}$ objetivo foi verificar se tais relações seriam transitivas, já que esses pares de estímulos não apareceram juntos na fase de ensino. Um bloco de revisão de linha de base com a apresentação dos cinco pares de ensino com critério de acerto de duas vezes consecutivas poderia preceder o teste nos casos de interrupção da sessão. $\mathrm{O}$ critério mínimo de desempenho de $80 \%$ de acerto (quatro pares) foi adotado nas fases de teste. Caso isso não fosse alcançado, o participante era reexposto à revisão. $\mathrm{O}$ mesmo foi realizado com estímulos do conjunto "A".

Por meio da estratégiadacomplementação, nas fases dos testes de transitividade e de substituição, a instrução inicial "agora você vai continuar fazendo como antes, mas os desenhos não irão aparecer" foi estendida e substituída por vários trechos no formato dialógico "sabe o joguinho que acabamos de fazer?" "então agora você vai fazer um pouco parecido, está bem?" "aqueles desenhos legais foram passear e voltam mais tarde!" "continue apontando um e depois o outro!" "agora olhe para as figuras!" "qual dessas vai primeiro?". O objetivo foi aumentar as chances de produzir os comportamentos esperados (Miccione, Carmo, \& Assis, 2013).

Teste de substituição com estímulos dos conjuntos "A" $\mathbf{e}$ "B". Após o ensino por sobreposição aos pares com os estímulos dos conjuntos "B" e "A", foram testados os pares adjacentes e não adjacentes entre sequências (cf. Stromer \& Mackay, 1993) em dois blocos com seis pares: A4 $\rightarrow$ B5, A3 $\rightarrow$ B4,
$\mathrm{B} 2 \rightarrow \mathrm{A} 5, \mathrm{~B} 3 \rightarrow \mathrm{A} 5, \mathrm{~A} 1 \rightarrow \mathrm{B} 2, \mathrm{~B} 2 \rightarrow \mathrm{A} 3-\mathrm{A} 2 \rightarrow \mathrm{B} 4$, $\mathrm{A} 3 \rightarrow \mathrm{B} 6, \mathrm{~B} 2 \rightarrow \mathrm{A} 6, \mathrm{~A} 3 \rightarrow \mathrm{B} 6, \mathrm{~B} 1 \rightarrow \mathrm{A} 2, \mathrm{~A} 1 \rightarrow \mathrm{B} 2 . \mathrm{O}$ objetivo foi verificar a emergência de classes ordinais por meio de tentativas com pares de estímulos de duas sequências diferentes. O critério mínimo de desempenho de $80 \%$ de acerto (quatro pares) foi adotado nas fases de teste. Caso isso não fosse alcançado, o participante era reexposto à revisão.

Para verificar os efeitos das fases de ensino, nas fases de teste cada tentativa foi apresentada uma única vez e sem nenhum tipo de consequenciação para as respostas.

\section{Etapa 2}

Ensino de sequências sob controle condicional auditivo. Antes de iniciar o ensino por sobreposição com a presença dos dois sons, houve uma fase anterior com o objetivo de apresentar aos participantes os novos estímulos sonoros. Seguindo-se esse direcionamento, a experimentadora os apresentava três vezes consecutivas de forma randômica. A experimentadora dizia "Eu vou mostrar pra você duas musiquinhas, está bem?". Após a apresentação do primeiro som era perguntado "Você ouviu?". Se afirmativo, o segundo som era apresentado. A experimentadora perguntava novamente se a criança tinha ouvido. Em caso afirmativo, esse mesmo procedimento era repetido por mais duas vezes. Se o participante respondesse não ter ouvido, o som era reapresentado até que a resposta fosse positiva.

Além dos estímulos utilizados na Etapa 1, dois sons com duração de 3 segundos cada. Um foi construído a partir de notas graves $\left(\mathrm{S}^{\mathrm{C}} x\right)$ e o outro com notas agudas $\left(\mathrm{S}^{\mathrm{C}} y\right)$. Cada tentativa iniciava com a apresentação randômica de um dos dois sons. A instrução fornecida era "Você lembra as musiquinhas que a gente ouviu? Elas vão tocar agora e você deverá selecionar uma figura e depois a outra" (a pesquisadora apontava a parte inferior da tela). A ativação do som (botão start, ver Figura 2) era realizada pela experimentadora após o consentimento do participante em iniciar a tentativa. Por exemplo, simultaneamente à apresentação do Som 1 , dois estímulos do Conjunto "B" eram apresentados e o participante deveria apontá-los na ordem crescente. Se emitido o Som 2, o participante deveria apontar na ordem inversa.

O critério de acerto era a formação correta de cada par de estímulos em três tentativas consecutivas, pri- 
meiramente na presença de um som e, em seguida, do outro som. O participante era exposto a um mesmo par no mínimo três vezes e, no máximo, 10. Após a sonda, o mesmo par era ensinado na ordem inversa. Em seguida, um novo par de estímulos era apresentado. Ao final um bloco misto de sondas de verificação com reforço apresentava os 10 pares ensinados por meio de dez tentativas randômicas. O critério desse bloco era de $80 \%$ de acertos (oito tentativas). Se não alcançado, um bloco de revisão de linha de base com critério de acerto de duas vezes consecutivas para cada par era apresentado. Esse mesmo bloco de revisão precedia a próxima fase de teste. $\mathrm{O}$ mesmo procedimento de ensino acima descrito foi adotado com os estímulos do Conjunto "A".

Teste de transitividade. Consistiu na apresentação de cinco pares não adjacentes intra-sequência sob as duas condições, sendo um tipo de bloco com os estímulos do Conjunto "B": B1 $\rightarrow$ A4, B4 $\rightarrow \mathrm{B} 1, \mathrm{~B} 5 \rightarrow \mathrm{B} 2$, $\mathrm{B} 2 \rightarrow \mathrm{B} 5, \quad \mathrm{~B} 4 \rightarrow \mathrm{B} 6, \quad \mathrm{~B} 6 \rightarrow \mathrm{B} 4, \quad \mathrm{~B} 1 \rightarrow \mathrm{B} 3, \quad \mathrm{~B} 3 \rightarrow \mathrm{B} 1$, $\mathrm{B} 2 \rightarrow \mathrm{B} 4, \mathrm{~B} 4 \rightarrow \mathrm{B} 2$; e outro com os estímulos do Conjunto "A": A1 $\rightarrow \mathrm{A} 5, \mathrm{~A} 5 \rightarrow \mathrm{A} 1, \mathrm{~A} 3 \rightarrow \mathrm{A} 1, \mathrm{~A} 1 \rightarrow \mathrm{A} 3$, $\mathrm{A} 3 \rightarrow \mathrm{A} 6, \mathrm{~A} 6 \rightarrow \mathrm{A} 3, \mathrm{~A} 4 \rightarrow \mathrm{A} 6, \mathrm{~A} 6 \rightarrow \mathrm{A} 4, \mathrm{~A} 2 \rightarrow \mathrm{A} 4$, $\mathrm{A} 4 \rightarrow \mathrm{A} 2$. O objetivo foi verificar se as relações ensinadas seriam transitivas sob controle condicional. Ao longo de um bloco, sempre uma tentativa era seguida do mesmo par após o critério de acerto, mas diante do outro estímulo condicional. Dessa forma, havia uma alternância quando da apresentação de um novo par de estímulos.

Dois blocos de revisão de linha de base, um para cada conjunto ("B" e "A") precederam o teste seguinte.
Teste de substituição com estímulos dos conjuntos "A" e "B". Foram testados os pares de estímulos adjacentes e não adjacentes entre sequências em um bloco com cinco pares sob as duas condições: $\mathrm{A} 3 \rightarrow \mathrm{B} 4, \quad \mathrm{~B} 4 \rightarrow \mathrm{A} 3, \quad \mathrm{~B} 1 \rightarrow \mathrm{A} 3, \quad \mathrm{~A} 3 \rightarrow \mathrm{B} 1, \quad \mathrm{~A} 6 \rightarrow \mathrm{B} 5$, $\mathrm{B} 5 \rightarrow \mathrm{A} 6, \mathrm{~A} 1 \rightarrow \mathrm{B} 5, \mathrm{~B} 5 \rightarrow \mathrm{A} 1, \mathrm{~A} 1 \rightarrow \mathrm{B} 2, \mathrm{~B} 2 \rightarrow \mathrm{A} 1$. O objetivo foi verificar a emergência de classes ordinais por meio de tentativas com pares de estímulos de duas sequências diferentes sob controle condicional. Assim como no teste de transitividade, o mesmo par de estímulos era apresentado na presença do outro estímulo condicional logo na tentativa seguinte, prevendo-se a condicionalidade dos sons.

Pós-testes. O conjunto de blocos composto pelo Pré-teste foi apresentado a todos os participantes da pesquisa. O objetivo foi verificar o efeito do procedimento sobre o repertório inicial dos participantes por meio da apresentação das mesmas tentativas.

\section{RESULTADOS}

A Tabela 1 demonstra os percentuais de acertos alcançados nas fases de ensino e de testes com os estímulos dos conjuntos "B" e "A", por participante. Todos alcançaram o critério de acerto de três vezes consecutivas para cada par de estímulos na fase de ensino, em ambos os Conjuntos. Num total de cinco pares de estímulos não adjacentes intra-sequência, todos apresentaram escores de acerto acima do nível do acaso com os pares do Conjunto B e $100 \%$ de acerto com os do A. O mesmo ocorreu com os pares no teste de Substituição.

\section{Tabela 1}

Percentuais de Acertos Alcançados nas Fases de Ensino e de Testes com os Estímulos dos Conjuntos "B" $e$ " $A$ ", por Participante

\begin{tabular}{cccc} 
& \multicolumn{3}{c}{ Participantes } \\
Fases & DGO & DNL & HLR \\
\hline Ensino de “B” & $*$ & $*$ & $*$ \\
Transitividade & $80^{* * *}$ & $60^{* * *}$ & $80^{* * *}$ \\
Ensino de “A" & $*$ & $*$ & $*$ \\
Transitividade & $100^{* *}$ & 100 & 100 \\
Substituição & $100^{* *}$ & 100 & 100 \\
\hline${ }^{*}$ Participante atingiu o critério de acerto nas sequências de ensino. \\
${ }^{* *}$ Desempenho alcançado na segunda exposição. \\
${ }^{* * *}$ Mesmo desempenho alcançado na primeira e na segunda exposição.
\end{tabular}


A Tabela 2 demonstra os percentuais de acertos alcançados nas fases de ensino e de testes na Etapa 2 com os estímulos dos Conjuntos "B e "A", por participante. Todos alcançaram o critério de acerto de três vezes consecutivas para cada par de estímulos na fase de ensino, em ambos os Conjuntos, sob as duas condições de estímulos sonoros. No teste de Transitividade
$\mathrm{B}$, todos alcançaram $60 \%$ de acertos nas tentativas. Em contrapartida, no teste de Transitividade A, $100 \%$ de acerto foi alcançado pelos participantes. Os resultados observados nos testes de Substituição demonstram que não houve formação de classes ordinais por nenhum participante.

\section{Tabela 2}

Percentuais de Acertos Alcançados nas Fases de Ensino e de Testes na Etapa 2 com os Estímulos dos Conjuntos "B e "A", por Participante

\begin{tabular}{lccc} 
Fases & \multicolumn{3}{c}{ Participantes } \\
& DGO & DNL & HLR \\
\hline Ensino de “B” & $*$ & $*$ & $*$ \\
Transitividade & 60 & 60 & 60 \\
Ensino de “A" & $*$ & $*$ & $*$ \\
Transitividade & $100^{* *}$ & 100 & 100 \\
Substituição & 50 & 50 & 50 \\
${ }^{*}$ Participante atingiu o critério de acerto nas sequências de ensino sob as \\
condições Som 1 e Som 2. \\
${ }^{* *}$ Desempenho apresentado na terceira exposição.
\end{tabular}

A Tabela 3 mostra que as porcentagens de acerto dos participantes nos Blocos do Pré-teste variaram de 29,16 a $50 \%$. A maior porcentagem corresponde ao escore máximo do critério de ingresso à pesquisa. Já no Bloco seguinte, as porcentagens de acerto dos participantes foi de 70,83 e 91,66, tendo-se, assim, um aumento de $29,17 \%, 41,67 \%$ e $41,66 \%$, respectivamente.

\section{Tabela 3 \\ Percentuais de Acertos Alcançados nos Blocos do Pré-teste e do Pós-teste, por Participante}

\begin{tabular}{c|ccc} 
& \multicolumn{3}{|c}{ Participantes } \\
& DGO & DNL & HLR \\
Pré-teste & 41,66 & 29,16 & 50 \\
Pós-teste & 70,83 & 70,83 & 91,66 \\
\hline
\end{tabular}

\section{DISCUSSÃO}

Todos os participantes alcançaram o critério de acerto nas sequências de ensino. Pode-se inferir que o tipo de procedimento produziu efeitos sobre a aprendizagem de ordenação aos pares, sob controle condicional. Por outro lado, os resultados nos testes de Transitividade com os estímulos do conjunto B e de Substituição sugerem que as relações formadas durante a fase de ensino restringiram-se ao critério de acerto, não apresentando, assim, as propriedades das relações ordinais.
Alguns autores discutem a dificuldade de instalação de repertório ordinal via sobreposição de pares adjacentes sobrepostos, tendo como base a inversão das funções ordinais e apresentação dos estímulos, respectivamente. Segundo eles, a mudança de função que os estímulos sofrem, tanto em contingências simples quanto em condicionais, podem comprometer a aprendizagem dessas relações (Almeida, 2000; Lopes Junior, \& Agostini, 2004; Martins \& Lopes Junior, 2000; Nunes \& Assis, 2006; Stromer \& Mackay, 1992a). De fato, a troca sistemática ao longo da apresentação da sequência pode configurar-se como pro- 
cessos sucessivos de reforçamento e extinção para o membro comum aos pares.

Um dos padrões de resposta analisado foi o controle por exclusão que os estímulos conhecidos exerceram sobre a resposta de ordenar quando apresentados juntos com estímulos desconhecidos no treino. A literatura sobre o responder por exclusão salienta que esse procedimento pode ser programado objetivando facilitar a aprendizagem (Mackay, Stoddard, \& Spencer, 1989). Essa mesma funçãopor exclusão pelo participante não foi observada neste estudo pelo fato dessa manipulação não ter sido planejada.

Adicionalmente, os estímulos condicionais auditivos programados para a presente pesquisa, uma vez apresentados, não permaneciam presentes durante a tentativa assim como descreve a literatura sobre o ensino por sobreposição de relações condicionais na modalidade visual-visual (Nunes \& Assis, 2006; Souza \& Assis, 2005; Souza et al., 2008). Da mesma forma como pode ocorrer no ambiente de sala de aula, onde o professor está presente fornecendo instruções ao aluno, pesquisas futuras poderiam investigar se a permanência dos estímulos auditivos durante toda a tentativa produziria o controle condicional sobre $\mathrm{o}$ desempenho dos participantes, favorecendo a formação de classes.

Assim como no Etapa 1, não houve efeito da ordem de apresentação dos Conjuntos sobre o desempenho no teste de Substituição. Ou seja, os participantes não apresentaram o padrão de selecionar primeiro o estímulo do Conjunto B e depois o do Conjunto A. Todavia, outro padrão foi encontrado. Todos os participantes responderam corretamente apenas aos pares cujos estímulos deveriam ser ordenados na ordem crescente, a mesma requerida na Etapa1. Com exceção das respostas ao par de estímulos $\mathrm{B} 3 \rightarrow \mathrm{B} 1$, os dados encontrados no teste de Transitividade B possibilitaram essa mesma análise.

Os dados apresentados permitem a elaboração das seguintes perguntas: Como não observar esses resultados se o treino sob controle condicional foi precedido pelo ensino aos pares apenas na ordem crescente? $\mathrm{O}$ treino em ambas as direções poderia garantir um menor número de tentativas sob contingências condicionais? Estaria o padrão de ensino presente em nossa cultura, o qual requer, em grande maioria, a organização dos eventos numa única direção, exercendo maior controle em detrimento do controle condicional previsto para esta pesquisa?

\section{CONSIDERAÇÕES FINAIS}

O objetivo deste trabalho foi investigar a produção de sequências com base na análise experimental das relações ordinais em crianças pré-escolares, utilizando-se estímulos nas modalidades visual-visual e visual-auditiva. Green et al., (1993) propuseram o paradigma das relações ordinais apresentando o procedimento por sobreposição de pares de estímulos. De acordo com esses autores, a investigação científica acerca do conceito de classes de estímulos, bem como a verificação das propriedades ordinais inerentes ao paradigma poderia ocorrer mais fidedignamente via sobreposição de estímulos, comparando-se ao procedimento por encadeamento.

Se por um lado o ensino por encadeamento pode favorecer a estabilidade da linha de base levando à formação de classes ordinais (relações entre sequências) com um número menor de erros ao produzido pelo procedimento por pares sobrepostos, por outro, o ensino por sobreposição pode possibilitar um controle experimental conspícuo sobre a relação entre produção de relações ordinais e avaliação de suas propriedades.

Sem desconsiderar a história extra-experimental simultânea à condução do experimento, a realização dos testes, bem como ao aumento no repertório dos participantes, permite a afirmação de que o procedimento foi eficaz em ensinar seis classes ordinais pela sobreposição de cinco pares de estímulos. Constatou-se que o aumento no número de sessões de treino aliado ao ajustamento das instruções ao tipo de população contribuíram para a redução no número de erros naquelas sessões (Miccione et al., 2012).

Dessa forma, os dados confirmam a proposição de Green et al., (1993) sobre a adequação das noções de cadeia e controle condicional à compreensão de repertórios ordinais, nos quais elos e membros podem ser identificados.

Complementarmente, a presente pesquisa subsidia a compreensão do comportamento simbólico na medida em que se programou o controle de estímulos na organização do delineamento. Compreende-se por comportamento simbólico, o a) responder a dois ou mais estímulos relacionados arbitrariamente; b) responder aos estímulos indiferenciadamente, assegurando-se a substituibilidade dos estímulos e c) responder aos estímulos diferencialmente em diferentes contextos.

Além disso, a condução da presente pesquisa possibilitou uma maior compreensão do procedimento 
por sobreposição de pares de estímulos em conjunto com controle condicional na modalidade auditivovisual não evidenciado experimentalmente, embora haja resultados positivos nos testes de transitividade com numerais cardinais (testes de transitividade A), mas não nos de substituibilidade. Ademais, pode corroborar os resultados já obtidos em estudos sobre relações ordinais emergentes e trazer contribuições relevantes para a compreensão das variáveis das quais o responder ordinal pode ser função, como no treino de cópia (visual-visual) e de ditado (som-visual) nas atividades acadêmicas de leitura e matemática (Miccione, Assis, \& Costa, 2010).

A produtividade do modo de investigação do responder ordinal aqui utilizado fornece uma abordagem empírica na medida em que subsidia o desenvolvimento e aplicação de tecnologias de ensino não somente da matemática, mas também de comportamentos complexos cuja ordem é a variável relevante como os gramaticais, de leitura e escrita e outros "simbólicos sequenciais", como sequência alfabética, espectro de cores, dias da semana e calendário com os meses do ano (por exemplo, Assis, Élleres, \& Sampaio, 2006; Green, 1991; Resende, Elias, \& Goyos, 2012; Ribeiro, Assis, \& Enumo, 2005; Sella \& Bandini, 2012; Skinner, 1957/1957).

Por fim, os resultados aqui obtidos oferecem questões para pesquisas futuras que considerem as análises apontadas ao longo das discussões, permitindo replicações sistemáticas que investiguem, pelo menos, duas variáveis: presença simultânea dos estímulos auditivos durante as tentativas e o esvanecimento dos estímulos a serem selecionados primeiramente.

\section{REFERÊNCIAS}

Almeida, A. C. (2000). Emergência de relações ordinais em crianças com baixo aproveitamento acadêmico. Dissertação de Mestrado, Programa de Pós-Graduação em Educação Especial, Universidade Federal de São Carlos, São Carlos, SP, Brasil.

Assis, G. J. A., \& Santos, M. B. (2010). PROLER (software - sistema computadorizado para o ensino de comportamentos conceituais). Belém, PA: Universidade Federal do Pará.

Assis, G., J. A., Corrêa, D. R., Souza, C. B. A., \& Prado, P. S. T. (2010). Aprendizagem de relações ordinais por meio de treino de uma única sequência de estímulos. Psicologia: Teoria e Pesquisa, 26, 675-685. doi: 10.1590/S0102-37722010000400011
Assis, G. J. A., Élleres, C. F., \& Sampaio, M. E. C. (2006). Emergência de relações sintáticas em crianças préescolares. Interação em Psicologia, 10, 19-29. doi: 10.5380/psi.v10i1.5786

Assis, G. J. A., Magalhães, P. G S., Monteiro, P. D. S., \& Carmo, J. S. (2011). Efeitos da ordem de ensino e da transferência de funções sobre relações ordinais em surdos. Acta Comportamentalia, 19, 43-63.

Bancroft, S. L., Weiss, J. S., Libby, M. E., \& Ahearn, W. H. (2011). A comparison of procedural variations in teaching behavior chains: Manual guidance, trainer completion, and no completion of untrained steps. Journal of Applied Behavior Analysis, 44, 559-569. doi:10.1901/ jaba.2011.44-559

Green, G. (1990). Differences in development of visual and auditory-visual equivalence relations. American Journal on Mental Retardation, 95, 260-270.

Green, G., Sigurdardottir, Z. G., \& Saunders, R. R. (1991). The role of instructions in the transfer of ordinal functions through equivalence classes. Journal of the Experimental Analysis of Behavior, 55, 287-304. doi: 10.1901/ jeab.1991.55-287

Green, G., Stromer, R., \& Mackay, H. A. (1993). Relational learning in stimulus sequences. The Psychological Record, 43, 599-616.

Holcomb, W. L., Stromer, R., \& Mackay, H. A. (1997). Transitivity and emergent sequence performance in young children. Journal of Experimental Child Psychology, 65, 96-124. doi: 10.1006/jecp.1996.2360

Hur, J., \& Osborne, S. (1993). A comparison of forward and backward chaining methods used in teaching corsage making skills to mentally retarded adults. The British Journal of Developmental Disabilities, 39, 108-117. doi: 10.1179/bjdd.1993.013

Lopes Júnior, J., \& Agostini, M. F. (2004). Aquisição e emergência de relações ordinais por crianças da segunda série do ensino fundamental. Em: O. M. P. R. Rodrigues, T. G. M. do Valle, A. C. M. Almeida-Verdu, J. Lopes Jr., M. R. Cavalcante (Eds.) Psicologia do desenvolvimento e aprendizagem: Investigações e análises (pp. 95127). São Carlos: Rima.

Mackay, H. A., Stoddard, L. T., \& Spencer, T. J. (1989). Symbols and meaning classes: Multiple sequence production and the emergence of ordinal stimulus classes. Experimental Analysis of Human BehaviorBulletin, 7, 16-17.

Martins, C., \& Lopes Júnior, J. (2000). Controle condicional sobre responder seqüencial por crianças com dificuldade de aprendizagem. Resumos de Comunicações Científicas da Reunião Anual da Sociedade Brasileira de Psicologia, Brasília, DF, Brasil.

Miccione, M. M., Assis, G., \& Carmo, J. S. (2012). Produção de sequências com base na análise experimental das relações ordinais em pré-escolares. Resumos de Comuni- 
cações Científicas da Associação Brasileira de Psicologia e Medicina Comportamental, Curitiba, PR, Brasil.

Miccione, M., Assis, G., \& Costa, T. D. (2010). Variáveis de controle sobre o responder ordinal: Revisitando estudos empíricos. Perspectivas em Análise do Comportamento, $1,130-148$.

Miccione, M. M., Carmo, J. S., \& Assis, G. (2013). Aprendendo a formar participantes colaborativos em pesquisa empírica: algumas considerações sobre variáveis metodológicas em sessões de coleta de dados. Comportamento em Foco, 2, 93-104.

Nunes, A. L. M., \& Assis, G. J. A. (2006). Emergência de classes ordinais após o ensino de relações numéricas. Revista Brasileira de Análise do Comportamento, 2, 203-219.

Resende, A. A. C., Elias, N. C. E., \& Goyos, C. (2012). Transferência de funções ordinais através de classes de estímulos equivalentes em surdos. Acta Comportamentalia, 20, 317-326.

Ribeiro, M. P. L., Assis, G. J. A., \& Enumo, S. R. F. (2005). Controle do comportamento por relações ordinais: questões conceituais e metodológicas. In E. B. Borloti, M. L. P. Ribeiro, \& S. R. F. Enumo (Orgs.). Análise do comportamento: teorias e práticas (pp. 117132). Santo André: ESETec.

Sella, A. C., \& Bandini, C. S. M. (2012). Aquisição, manutenção e generalização de sequências verbais: Alguns contrapontos entre a Análise do Comportamento e Abordagens Cognitivistas. Acta Comportamentalia, 20, 157176.

Sidman, M., \& Tailby, W. (1982). Conditional discrimination versus matching to sample: an expansion of the testing paradigm. Journal of the Experimental Analysis of Behavior, 37, 5-22. doi: 10.1901/jeab.1982.37-5

Sidman, M., Wilson-Morris, M., \& Kirk, B. (1986). Matching-to-sample procedures and the development of equivalence relations: the role of naming. Analysis and Intervention in Developmental Disabilities, 6, 1-19. doi: 10.1016/0270-4684(86)90003-0

Skinner, B. F. (1991). The behavior of organisms: An experimental analysis. Acton (MA): Copley Publishing Group (originalmente publicado em 1938).
Skinner, B. F. (1992). Verbal behavior. Acton (MA): Copley Publishing Group (originalmente publicado em 1957).

Smeets, P. M., \& Barnes-Holmes, D. (2005). Auditoryvisual and visual-visual equivalence relations in children. The Psychological Record, 55, 483-503.

Souza, R. D. C., \& Assis, G. J. A. (2005). Emergência de relações ordinais em crianças surdas. Psicologia: Teoria e Pesquisa, 21, 297-308.

Souza, R. D. C., Assis, G. J. A., \& Magalhães, P. G. S. (2005). Equivalência numérica em crianças surdas. Temas em Psicologia. 13, 113-127.

Souza, R. D. C., Assis, G. J. A., Magalhães, P. G. S., \& Prado, P. S. T. (2008). Emergência de relações numéricas sob controle condicional em surdos. Interação em Psicologia, 12, 59-75.

Souza, R. D. C., Magalhães, P. G. S., Assis, G., \& Goulart, P. R. K. (2010). Emergência de relações ordinais sob controle contextual em surdos. Revista Brasileira de Análise do Comportamento, 6, 37-54.

Spradlin, J. E. (1993). Rotinas: implicações para a vida e para o ensino. Temas em Psicologia, 7, 223-234.

Staats, A. W., \& Staats, C. K. (1963). Complex human behavior: A systematic extension of learning principles. Michigan: Holt, Rinehart \& Winston.

Stevens, J. (1951). Mathematics, measurement, and psychophysics. In S. Stevens (Ed.), Handbook of experimental psychology (pp. 1-49). New York: John Wiley and Sons, Inc.

Stromer, R. \& Mackay, H. A. (1992a). Conditional stimulus control of children's sequence production. Psychological Reports, 70, 903-912.

Stromer, R., \& Mackay, H. A. (1993). Human sequential behavior: relations among stimuli, class formation and derived sequences. The Psychological Record, 43, 107131.

Verdu, A. C. M. A., Souza, D. G., \& Lopes Júnior, J. L. (2005). Formação de classes ordinais após a aprendizagem de sequências independentes. Estudos de Psicologia, 11, 87-99.

Submetido em: 24/03/2013

Primeira decisão editorial em: 09/11/2014

Aceito em: 20/12/2014

\section{Nota:}

1 Segundo Green et al. (1993), na referida notação alfanumérica, a letra corresponde ao tipo de estímulo (classe); o número, à posição do estímulo na sequência, e a seta indica a direção da relação entre os estímulos. 